\title{
On a Class of Multiattribute Utility Functions Invariant under Shift Transformations
}

\author{
J. ChudziaK* \\ Department of Mathematics, University of Rzeszów, Rejtana 16 C \\ 35-959 Rzeszów, Poland
}

\begin{abstract}
We consider a class of multiattribute utility functions which are invariant with respect to the shifts having identical parameters for each attribute.
\end{abstract}

PACS numbers: 89.65.Gh

\section{Introduction}

Following [1, Definition 2, p. 19], we say that a utility function $U$ is invariant to a continuous transformation $g$, provided it satisfies the following functional equation

$$
U(g(x, z))=k(z) U(x)+\ell(z),
$$

with some functions $k$ and $\ell$. If $g$ is a shift transformation, that is $g(x, z)=x+z$, Eq. (1) reduces to

$$
U(x+z)=k(z) U(x)+\ell(z)
$$

and a utility function $U$ satisfying (2) is said to be invariant under shift transformation. Some results concerning utility functions invariant under shift transformation could be found e.g. in [1] and [3-5]. In a recent paper [2] this notion has been extended into the case of $n$-attribute utility functions. The problem in a natural way leads to the following generalization of (2)

$$
\begin{aligned}
& U\left(x_{1}+z_{1}, \ldots, x_{n}+z_{n}\right) \\
& \quad=k\left(z_{1}, \ldots, z_{n}\right) U\left(x_{1}, \ldots, x_{n}\right)+\ell\left(z_{1}, \ldots, z_{n}\right) .
\end{aligned}
$$

It turns out that in many cases (e.g. if the initial wealth of the decision maker is in the form of annuity payment which pays an amount $z$ at every period for $n$ successive periods) it is reasonable to assume that the utility function satisfies invariance just when the shift parameters are identical for each attribute, i.e. $z_{1}=\ldots=z_{n}=z$ with $z$ in an interval of positive length. It is clear that in such a case Eq. (3) reduces to

$$
\begin{aligned}
& U\left(x_{1}+z, \ldots, x_{n}+z\right) \\
& \quad=k(z) U\left(x_{1}, \ldots, x_{n}\right)+\ell(z) .
\end{aligned}
$$

Equation (4) has been already solved in [2] under the assumptions that $D$ is a non-empty open set, for every $\left(x_{1}, \ldots, x_{n}\right) \in D$, the set $V_{\left(x_{1}, \ldots, x_{n}\right)}:=\left\{z \in \mathbb{R} \mid\left(x_{1}+z, \ldots, x_{n}+z\right) \in D\right\}$ is an interval, $U: D \rightarrow \mathbb{R}, k, \ell: V_{\mathrm{D}}:=\bigcup_{\left(x_{1}, \ldots, x_{n}\right) \in D} V_{\left(x_{1}, \ldots, x_{n}\right)} \rightarrow \mathbb{R}$ are unknown functions, and a function

\footnotetext{
* e-mail: chudziak@univ.rzeszow.pl
}

$$
V_{\left(x_{1}, \ldots, x_{n}\right)} \ni z \rightarrow U\left(x_{1}+z, \ldots, x_{n}+z\right)
$$

is non-constant for at least one $\left(x_{1}, \ldots, x_{n}\right) \in D$. However, it is easy to check that $[2$, Theorem 4.3, p. 9] remains true if, instead of the openness of $D$ we assume that, for every $\left(x_{1}, \ldots, x_{n}\right) \in D$, the set $V_{\left(x_{1}, \ldots, x_{n}\right)}$ is an open interval. In order to formulate that result in such a modified version, we need to introduce the following notation. Let

$$
T:=\left\{\left(x_{2}-x_{1}, \ldots, x_{n}-x_{1}\right) \mid\left(x_{1}, \ldots, x_{n}\right) \in D\right\}
$$

and, for every $\left(t_{1}, \ldots, t_{n-1}\right) \in T$,

$$
V^{\left(t_{1}, \ldots, t_{n-1}\right)}:=\bigcup_{\left(x_{1}, \ldots, x_{n}\right) \in D,\left(x_{2}-x_{1}, \ldots, x_{n}-x_{1}\right)=\left(t_{1}, \ldots, t_{n-1}\right)} V_{\left(x_{1}, \ldots, x_{n}\right)} .
$$

Furthermore, given a function $\psi: T \rightarrow \mathbb{R}$, we set

$$
V_{\psi \neq 0}:=\bigcup_{\left(x_{1}, \ldots, x_{n}\right) \in D, \psi\left(x_{2}-x_{1}, \ldots, x_{n}-x_{1}\right) \neq 0} V_{\left(x_{1}, \ldots, x_{n}\right)} .
$$

Theorem 1. Let $D$ be a nonempty subset of $\mathbb{R}^{n}$ such that, for every $\left(x_{1}, \ldots, x_{n}\right) \in D, V_{\left(x_{1}, \ldots, x_{n}\right)}$ is an open interval. Assume that $U: D \rightarrow \mathbb{R}, k, \ell: V_{\mathrm{D}} \rightarrow \mathbb{R}$ and a function given by (5) is non-constant for at least one $\left(x_{1}, \ldots, x_{n}\right) \in D$. Then a triple $(U, k, \ell)$ satisfies $E q$. (4) for all $\left(x_{1}, \ldots, x_{n}\right) \in D$ and $z \in V_{\left(x_{1}, \ldots, x_{n}\right)}$ if and only if one of the subsequent two conditions holds:

(i) there exist a nonconstant additive function $a: \mathbb{R} \rightarrow$ $\mathbb{R}$ and a function $\psi: T \rightarrow \mathbb{R}$ such that

$$
\left\{\begin{array}{lr}
k(z)=1 & \text { for } z \in V_{\mathrm{D}} \\
\ell(z)=a(z) & \text { for } z \in V_{\mathrm{D}} \\
U\left(x_{1}, \ldots, x_{n}\right)=\psi\left(x_{2}-x_{1}, \ldots, x_{n}-x_{1}\right) & \\
+a\left(x_{1}\right) & \text { for }\left(x_{1}, \ldots, x_{n}\right) \in D ;
\end{array}\right.
$$

(ii) there exist a nonconstant exponential function $e$ : $\mathbb{R} \rightarrow \mathbb{R}$, a constant $c \in \mathbb{R}$ and a not identically zero function $\psi: T \rightarrow \mathbb{R}$ such that 


$$
\left\{\begin{array}{lr}
k(z)=e(z) & \text { for } z \in V_{\psi \neq 0} \\
\ell(z)=c(1-k(z)) & \text { for } z \in V_{\mathrm{D}} \\
U\left(x_{1}, \ldots, x_{n}\right)=e\left(x_{1}\right) \psi\left(x_{2}-x_{1}, \ldots, x_{n}-x_{1}\right) \\
+c & \text { for }\left(x_{1}, \ldots, x_{n}\right) \in D .
\end{array}\right.
$$

The aim of the present paper is to complete the results of [2] by determining all solutions of (4) such that a function given by $(5)$ is constant for every $\left(x_{1}, \ldots, x_{n}\right) \in D$.

\section{Results}

We begin this section with the following remark Remark 1. Since, for every $\left(x_{1}, \ldots, x_{n}\right) \in D, V_{\left(x_{1}, \ldots, x_{n}\right)}$ is an open interval containing 0 , it is clear that, for every $\left(t_{1}, \ldots, t_{n-1}\right) \in T, V^{\left(t_{1}, \ldots, t_{n-1}\right)}$ is also an open interval containing 0. Moreover, for every $\left(t_{1}, \ldots, t_{n-1}\right) \in T$, $V^{\left(t_{1}, \ldots, t_{n-1}\right)}$ is symmetric with respect to 0 . In fact, if $z \in$ $V^{\left(t_{1}, \ldots, t_{n-1}\right)}$ then $z \in V_{\left(x_{1}, \ldots, x_{n}\right)}$ for some $\left(x_{1}, \ldots, x_{n}\right) \in$ $D$ with $\left(x_{2}-x_{1}, \ldots, x_{n}-x_{1}\right)=\left(t_{1}, \ldots, t_{n-1}\right)$. Thus, $\left(x_{1}+z, \ldots, x_{n}+z\right) \in D$, whence $-z \in V_{\left(x_{1}+z, \ldots, x_{n}+z\right)}$. As $\left(x_{2}+z-\left(x_{1}+z\right), \ldots, x_{n}+z-\left(x_{1}+z\right)\right)=\left(t_{1}, \ldots, t_{n-1}\right)$, this means that $-z \in V^{\left(t_{1}, \ldots, t_{n-1}\right)}$.

The next theorem is a main result of the paper

Theorem 2. Let $D$ be a nonempty subset of $\mathbb{R}^{n}$ such that, for every $\left(x_{1}, \ldots, x_{n}\right) \in D, V_{\left(x_{1}, \ldots, x_{n}\right)}$ is an open interval. Assume that $U: D \rightarrow \mathbb{R}, k, \ell: V_{\mathrm{D}} \rightarrow$ $\mathbb{R}$ and a function given by (5) is constant for every $\left(x_{1}, \ldots, x_{n}\right) \in D$. Then a triple $(U, k, \ell)$ satisfies Eq. (4) for all $\left(x_{1}, \ldots, x_{n}\right) \in D$ and $z \in V_{\left(x_{1}, \ldots, x_{n}\right)}$ if and only if there exist a constant $d \in \mathbb{R}$ and a function $\psi: T \rightarrow \mathbb{R}$ such that

$$
\begin{aligned}
& \psi\left(t_{1}, \ldots, t_{n-1}\right)=d \\
& \text { whenever } V^{\left(t_{1}, \ldots, t_{n-1}\right)} \backslash k^{-1}(\{1\}) \neq \emptyset, \\
& \ell(z)=d(1-k(z)) \text { for } z \in V_{\mathrm{D}}
\end{aligned}
$$

and

$$
\begin{aligned}
& U\left(x_{1}, \ldots, x_{n}\right)=\psi\left(x_{2}-x_{1}, \ldots, x_{n}-x_{1}\right) \\
& \quad \text { for } \quad\left(x_{1}, \ldots, x_{n}\right) \in D .
\end{aligned}
$$

Prof: In order to prove that (8) holds with some function $\psi: T \rightarrow \mathbb{R}$ it is enough to show that, for every $\left(x_{1}, \ldots, x_{n}\right),\left(y_{1}, \ldots, y_{n}\right) \in D$, the following implication is valid:

$$
\begin{aligned}
& \left(x_{2}-x_{1}, \ldots, x_{n}-x_{1}\right)=\left(y_{2}-y_{1}, \ldots, y_{n}-y_{1}\right) \\
& \Longrightarrow U\left(x_{1}, \ldots, x_{n}\right)=U\left(y_{1}, \ldots, y_{n}\right) .
\end{aligned}
$$

To this end, fix $\left(x_{1}, \ldots, x_{n}\right),\left(y_{1}, \ldots, y_{n}\right) \in D$ and suppose that $\left(x_{2}-x_{1}, \ldots, x_{n}-x_{1}\right)=\left(y_{2}-y_{1}, \ldots, y_{n}-y_{1}\right)$. Then $y_{j}=x_{j}+\left(y_{1}-x_{1}\right)$ for $j=1,2, \ldots, n$ and so, using the fact that a function given by (5) is constant for $\left(x_{1}, \ldots, x_{n}\right)$, we get

$$
\begin{aligned}
& U\left(y_{1}, \ldots, y_{n}\right)=U\left(x_{1}+\left(y_{1}-x_{1}\right), \ldots, x_{n}+\left(y_{1}-x_{1}\right)\right) \\
& \quad=U\left(x_{1}, \ldots, x_{n}\right) .
\end{aligned}
$$

Therefore, (8) is proved. Now, note that as a function given by $(5)$ is constant for every $\left(x_{1}, \ldots, x_{n}\right) \in D$, from (4) and (8) we derive that

$$
\begin{aligned}
& \ell(z)=(1-k(z)) \psi\left(x_{2}-x_{1}, \ldots, x_{n}-x_{1}\right) \\
& \quad \text { for } \quad\left(x_{1}, \ldots, x_{n}\right) \in D, z \in V^{\left(x_{2}-x_{1}, \ldots, x_{n}-x_{1}\right)} .
\end{aligned}
$$

Thus, if $k$ is identically 1 , then $\ell$ is identically 0 . Hence, taking an arbitrary $d \in \mathbb{R}$, we get the assertion. So, assume that $k$ is not identically 1 . Put

$$
V_{\mathrm{D}}^{+}:=\left\{z \in V_{\mathrm{D}} \cap[0, \infty) \mid k(z) \neq 1\right\}
$$

and

$$
V_{\mathrm{D}}^{-}:=\left\{z \in V_{\mathrm{D}} \cap(-\infty, 0) \mid k(z) \neq 1\right\} .
$$

Clearly, at least one of the sets $V_{\mathrm{D}}^{+}$and $V_{\mathrm{D}}^{-}$is nonempty. Assume that $V_{\mathrm{D}}^{+} \neq \emptyset$ and let $z_{0}^{+}:=\inf V_{\mathrm{D}}^{+}$. Let $\left\{z_{n}^{+}\right\}$be a sequence of elements of $V_{\mathrm{D}}^{+}$converging to $z_{0}^{+}$. Fix a $z \in V_{\mathrm{D}}^{+}$and $\left(\tilde{x_{1}}, \ldots, \tilde{x_{n}}\right) \in D$ with $z \in V_{\left(\tilde{x_{1}}, \ldots, \tilde{x_{n}}\right)}$. Since $z_{0}^{+} \leq z$ and $V_{\left(\tilde{x_{1}}, \ldots, \tilde{x_{n}}\right)}$ is an interval containing 0 , for sufficiently large $n \in \mathbb{N}$, we have $z_{n}^{+} \in V_{\left(\tilde{x_{1}}, \ldots, \tilde{x_{n}}\right)}$. Then, in view of (9), for sufficiently large $n \in \mathbb{N}$, we obtain

$$
\ell\left(z_{n}^{+}\right)=\left(1-k\left(z_{n}^{+}\right)\right) \psi\left(\tilde{x_{2}}-\tilde{x_{1}}, \ldots, \tilde{x_{n}}-\tilde{x_{1}}\right),
$$

whence

$$
\lim _{n \rightarrow \infty} \frac{\ell\left(z_{n}^{+}\right)}{1-k\left(z_{n}^{+}\right)}=\psi\left(\tilde{x_{2}}-\tilde{x_{1}}, \ldots, \tilde{x_{n}}-\tilde{x_{1}}\right)=: d .
$$

Next, taking an arbitrary $\left(x_{1}, \ldots, x_{n}\right) \in D$ such that $V_{\left(x_{1}, \ldots, x_{n}\right)} \cap V_{\mathrm{D}}^{+} \neq \emptyset$ and arguing as previously, we get that

$$
d=\lim _{n \rightarrow \infty} \frac{\ell\left(z_{n}^{+}\right)}{1-k\left(z_{n}^{+}\right)}=\psi\left(x_{2}-x_{1}, \ldots, x_{n}-x_{1}\right) .
$$

In this way we have proved that there is a $d \in \mathbb{R}$ such that

$$
\begin{aligned}
& V_{\left(x_{1}, \ldots, x_{n}\right)} \cap V_{\mathrm{D}}^{+} \neq \emptyset \\
& \quad \Longrightarrow \psi\left(x_{2}-x_{1}, \ldots, x_{n}-x_{1}\right)=d .
\end{aligned}
$$

Analogously, one can prove that, if $V_{\mathrm{D}}^{-} \neq \emptyset$, then there exists a $\tilde{d} \in \mathbb{R}$ such that

$$
\begin{aligned}
& V_{\left(x_{1}, \ldots, x_{n}\right)} \cap V_{\mathrm{D}}^{-} \neq \emptyset \\
& \quad \Longrightarrow \psi\left(x_{2}-x_{1}, \ldots, x_{n}-x_{1}\right)=\tilde{d} .
\end{aligned}
$$

Now, in order to show (6) it remains to prove that if both sets $V_{\mathrm{D}}^{+}$and $V_{\mathrm{D}}^{-}$are nonempty, then $d=\tilde{d}$. So, suppose that $V_{\mathrm{D}}^{+} \neq \emptyset$ and $V_{\mathrm{D}}^{-} \neq \emptyset$. Let $z_{0}^{-}:=\sup V_{\mathrm{D}}^{-}$and, as previously, $z_{0}^{+}:=\inf V_{\mathrm{D}}^{+}$. Assume that $z_{0}^{-} \geq-z_{0}^{+}$ (in the case where $z_{0}^{-} \leq-z_{0}^{+}$, the proof runs analogously). Then $0 \leq z_{0}^{-}+z_{0}^{+} \leq z_{0}^{+}$whence, taking $\left(x_{1}, \ldots, x_{n}\right) \in D$ such that $V_{\left(x_{1}, \ldots, x_{n}\right)} \cap V_{\mathrm{D}}^{+} \neq \emptyset$, we get $z_{0}^{-}+z_{0}^{+} \in V_{\left(x_{1}, \ldots, x_{n}\right)}$ and so $z_{0}^{-} \in V_{\left(x_{1}+z_{0}^{+}, \ldots, x_{n}+z_{0}^{+}\right)}$. Since $V_{\left(x_{1}+z_{0}^{+}, \ldots, x_{n}+z_{0}^{+}\right)}$is an open interval, this means that $V_{\left(x_{1}+z_{0}^{+}, \ldots, x_{n}+z_{0}^{+}\right)} \cap V_{\mathrm{D}}^{-} \neq \emptyset$. Thus, by (11), $\psi\left(x_{2}-x_{1}, \ldots, x_{n}-x_{1}\right)=\tilde{d}$. On the other hand, as $V_{\left(x_{1}, \ldots, x_{n}\right)} \cap V_{\mathrm{D}}^{+} \neq \emptyset$, by (10), we get $\psi\left(x_{2}-x_{1}, \ldots, x_{n}-x_{1}\right)=d$. Hence $d=\tilde{d}$ and so (6) is proved. Finally, we show that (7) holds. Since $k$ is not identically 1 , there is $z_{0} \in V_{\mathrm{D}}$ with $k\left(z_{0}\right) \neq 1$. Let 
$\left(x_{1}^{0}, \ldots, x_{n}^{0}\right) \in D$ be such that $z_{0} \in V^{\left(x_{2}^{0}-x_{1}^{0}, \ldots, x_{n}^{0}-x_{1}^{0}\right)}$. Then $V^{\left(x_{2}^{0}-x_{1}^{0}, \ldots, x_{n}^{0}-x_{1}^{0}\right)} \backslash k^{-1}(\{1\}) \neq \emptyset$ and so, in view of $(6)$, we get $\psi\left(x_{2}^{0}-x_{1}^{0}, \ldots, x_{n}^{0}-x_{1}^{0}\right)=d$. Thus, making use of (9), we conclude that

$$
\ell(z)=d(1-k(z)) \quad \text { for } \quad z \in V^{\left(x_{2}^{0}-x_{1}^{0}, \ldots, x_{n}^{0}-x_{1}^{0}\right)} .
$$

Now, let $z \in V_{\mathrm{D}}$ be arbitrary and $\left(x_{1}, \ldots, x_{n}\right) \in D$ be such that $z \in V^{\left(x_{2}-x_{1}, \ldots, x_{n}-x_{1}\right)}$. According to Remark $1, \quad V^{\left(x_{2}^{0}-x_{1}^{0}, \ldots, x_{n}^{0}-x_{1}^{0}\right)}$ and $V^{\left(x_{2}-x_{1}, \ldots, x_{n}-x_{1}\right)}$ are intervals symmetric with respect to 0 . Thus, either $V^{\left(x_{2}-x_{1}, \ldots, x_{n}-x_{1}\right)} \subset V^{\left(x_{2}^{0}-x_{1}^{0}, \ldots, x_{n}^{0}-x_{1}^{0}\right)}$ or $V^{\left(x_{2}^{0}-x_{1}^{0}, \ldots, x_{n}^{0}-x_{1}^{0}\right)} \subset V^{\left(x_{2}-x_{1}, \ldots, x_{n}-x_{1}\right)}$. In the first case, in view of (12), we get $\ell(z)=d(1-k(z))$. In the second one, we have $V^{\left(x_{2}-x_{1}, \ldots, x_{n}-x_{1}\right)} \backslash k^{-1}(\{1\}) \neq \emptyset$ which, together with (6) and (9), gives again $\ell(z)=d(1-k(z))$. Therefore (7) holds and the proof is completed.

Example 1. Let $D=D_{1} \cup D_{2}$, where

$$
D_{1}=\left\{\left(x_{1}, x_{2}\right) \in \mathbb{R}^{2} \mid 1 \leq x_{2}-x_{1}<2,1<x_{1}+x_{2}<2\right\}
$$
and

$$
D_{2}=\left\{\left(x_{1}, x_{2}\right) \in \mathbb{R}^{2} \mid 0<x_{2}-x_{1}<1,1<x_{1}+x_{2}<3\right\} .
$$

Then, for every $\left(x_{1}, x_{2}\right) \in D$, the set $V_{\left(x_{1}, x_{2}\right)}$ is an open interval. In fact, we have

$$
V_{\left(x_{1}, x_{2}\right)}= \begin{cases}\left(\frac{1-\left(x_{1}+x_{2}\right)}{2}, \frac{2-\left(x_{1}+x_{2}\right)}{2}\right) & \text { for }\left(x_{1}, x_{2}\right) \in D_{1} \\ \left(\frac{1-\left(x_{1}+x_{2}\right)}{2}, \frac{3-\left(x_{1}+x_{2}\right)}{2}\right) & \text { for }\left(x_{1}, x_{2}\right) \in D_{2} .\end{cases}
$$

Thus $V_{\mathrm{D}}=(-1,1)$. Furthermore, we have $T=(0,2)$ and

$$
V^{t}=\left\{\begin{array}{l}
(-1,1) \text { for } t \in(0,1) \\
\left(-\frac{1}{2}, \frac{1}{2}\right) \text { for } t \in[1,2) .
\end{array}\right.
$$

Fix $d \in \mathbb{R}$ and $\Delta \in[0, \infty)$. Let $k:(-1,1) \rightarrow \mathbb{R}$ be an arbitrary function such that $k^{-1}(\{1\})=[-\Delta, \Delta]$ and let $\ell(z)=d(1-k(z))$ for $z \in(-1,1)$. Note that:

- if $\Delta \geq 1$ then $V^{t} \backslash k^{-1}(\{1\})=\emptyset$ for every $t \in(0,2)$;

- if $\Delta \in\left[\frac{1}{2}, 1\right)$ then $V^{t} \backslash k^{-1}(\{1\}) \neq \emptyset$ for every $t \in(0,1)$;
- if $\Delta \in\left[0, \frac{1}{2}\right)$ then $V^{t} \backslash k^{-1}(\{1\}) \neq \emptyset$ for every $t \in(0,2)$.

Therefore, applying Theorem 2, we conclude that a triple $(k, \ell, U)$, where $U: D \rightarrow \mathbb{R}$, satisfies (4) if and only if

$$
U\left(x_{1}, x_{2}\right)=\psi\left(x_{2}-x_{1}\right) \quad \text { for } \quad\left(x_{1}, x_{2}\right) \in D,
$$

where $\psi:(0,2) \rightarrow \mathbb{R}$ is such that:

a) $\psi_{\mid(0,1)} \equiv d$ in the case where $\Delta \in\left[\frac{1}{2}, 1\right)$;

b) $\psi \equiv d$ in the case where $\Delta \in\left[0, \frac{1}{2}\right)$.

\section{Conclusion}

In several cases, the initial wealth of the decision maker is in the form of annuity payment which pays an amount $z$ at every period for $n$ successive periods. In such cases, a multiattribute utility function is invariant under shift transformation, provided Eq. (2) holds with some functions $k$ and $\ell$. This equation has been considered in [2] under the assumption that a function given by (5) is non-constant for at least one $\left(x_{1}, \ldots, x_{n}\right) \in D$. In the present paper we complete the results in [2] by determining the functional form of utility function in the case where a function given by (5) is constant for every $\left(x_{1}, \ldots, x_{n}\right) \in D$.

\section{References}

[1] A.E. Abbas, Decision Analysis 4, 17 (2007).

[2] A.E. Abbas, J. Aczél, J. Chudziak, Result. Math. 54, 1 (2009).

[3] R.A. Howard, IEEE Trans. Systems Sci. Cybernet. 3, 24 (1967).

[4] J. Pfanzagl, Naval Research Logistic Quarterly 6, 283 (1959).

[5] H. Raiffa, Decision Analysis, Addison-Wesley, Reading, Massachusetts 1968. 